\title{
Optimization of the Energy Consumption of Connected Objects
}

\author{
https://doi.org/10.3991/ijim.v15i24.26985 \\ Mohammed Moutaib ${ }^{1(凶)}$, Tarik Ahajjam ${ }^{1}$, Mohammed Fattah ${ }^{1}$, Yousef Farhaoui ${ }^{1}$, \\ Badraddine Aghoutane ${ }^{1}$, Moulhime El Bekkali \\ ${ }^{1}$ University of Moulay Ismail, Meknes, Morocco \\ ${ }^{2}$ Sidi Mohamed Ben Abdellah University, Fez, Morocco \\ mohammed.93@live.fr
}

\begin{abstract}
The IoT is a growing new approach that has been defined as a global network of devices and machines capable of reliably communicating with each other without human intervention. It is one of the essential technologies in any field, such as medicine and attracts great attention in the future. It is applied in several areas that have achieved success. However, the power and the addition of connected objects to technology is based on the fact that its objects can establish several tasks: communicate, analyze, process and manage data in a parallel manner, which is very difficult in terms of energy consumption. Therefore, the problems related to consumption slow down considerably the evolution and the fast deployment of this high technology. Therefore, it is necessary to create a new lightweight and robust mechanism, which ensures the minimization of the consumption of the objects and makes these objects efficient and less costly while being adapted to the capacities of objects and technologies. That is why our paper aims to address this significant problem and present the role of energy consumption, which is essential in deploying successful IoT products and services and presenting the IoT categories for applications. First, we propose a method that minimizes energy consumption and meets our need through three essential steps: firstly, to study the existing methods to minimize energy consumption. Next, based on these methods, we create a new concept using the data flows. Finally, we implement our solution in an intelligent parking lot to carry out our approach and describe our design steps and conclude with the result of our study and make an interpretation that summarizes our work.
\end{abstract}

Keywords-IOT, cloud computing, RFID, WSN

\section{Introduction}

The IoT is a notion that has developed ubiquitous computing [1], in which technology gradually disappears into the user's environment and is naturally integrated into everyday objects. This technology represents several objects in the form of a dedicated and easy-to-use device that can communicate on several types of wireless networks. 
In addition, the IoT Cultivates a large number of new applications, for example, Environmental monitoring, health care and efficient energy management in smart homes [2], potentially generating significant economic benefits [3]. In fact, the US estimates that the Internet of Things is one of six Civil technology can have an impact on international power [4].

A Connected Object can interact independently with our world without any intervention. It has some limitations, such as storage, memory or power consumption. It needs to be adopted, it has some form of intelligence and can transmit and receive data through sensors [5]. Connected objects have value when they are connected to other objects and software.

Indeed, the IoT has currently reflected in the number of connected objects with their own identities and increasingly complex computing and communication capabilities: phones, watches, household appliances and other objects equipped with more and more sensors and Actuators, allowing them to measure and act on the environment, thus establishing a connection between the physical and virtual worlds.

Therefore, in terms of architecture, the concept of the IoT Aspects, protocol stacks, applications and conceptual vision, research has recently begun [6-7]. Smart grids are considered one of IoT's main applications and have attracted a lot of interest in recent years [2, 8-9].

In addition, the European technology platform The Intelligent System Integration (EPoSS) defines IoT as a global network with uniquely addressable interconnected objects, Based on the standard communication protocol [10].

As IoT technology advances and more businesses embrace it, IoT analytics is transforming as a topic of great value. Thanks to these vast advantages, companies are looking for high investment in IoT. However, despite this advantage, they must carefully evaluate every opportunity and challenge induced by the IoT to ensure that their resources are spent reasonably.

The Internet of Things poses several challenges, with its great benefit, dynamic nature, and heterogeneous data and systems that consist of powerful devices and various other advantages like mobility, power, etc... These characteristics require tools and methods to realize applications, extract information and data from several available data sources, store and archive them in databases.

On the other hand, connected objects oriented towards comfort or leisure, be it automation processes or entertainment tools. Moreover, this is the paradox of this revolution of connected objects: this connectivity requires energy, much energy. Also, for the time being, the savings made by the objects that are virtuous from this point of view do not compensate the additional energy expenditure induced by all the connected objects equipping homes.

These energy costs are divided between battery-powered devices that need to be recharged regularly, constant-power devices (refrigerators, televisions, washing machines, thermostats, etc.), and the servers and routers needed to ensure the connectivity of the objects.

As for battery-powered connected objects, it is evident that a sharp decline in autonomy accompanies their technological progress. Performance can be improved, but the need to maintain a connection to the Internet or the local network is an energy drain. 
Just as the autonomies of phones melted like snow in the sun when they became smart, those of connected objects are often highly reduced:

- a connected vacuum cleaner doesn't exceed 45 minutes of autonomy, Anki's Cozmo robot or Samsung Gear IconX headphones struggle to reach an hour and a half without being reconnected.

The problem with a connected object is that it works continuously to produce data and transmit it in real-time: it must respond continuously, even when not in use. Maintaining this connectivity to the network consumes on average $80 \%$ of the energy spent by a device:

As a result, connected devices consume almost as much activity or standby in their majority. For example, a connected TV consumes on average $30 \mathrm{~W}$ when it is on and $25 \mathrm{~W}$ when it is on standby!

Some researchers speak of hyper objects [11] capable of pooling their resources to perform complicated tasks, links within the same network link these objects. Other researchers like [12] have considered computing as one of the most profound technologies. This technology makes this technology the fabric of our daily life to becoming inseparable [13].

In this context, we propose a solution that minimizes energy consumption. Our solution is based on IoT technologies. In fact, our approach is composed of several axes from the definition to the result.

We start by schematizing the solution in the first part of our solution and implement it in intelligent parking to measure the effectiveness of our proposal on different axes.

The rest of this paper is organized like this. Section 2 presents the application of the Internet of Things in the various existing fields. In section 3, we will present some IOT technologies. We will list some related works in section 4 . In the penultimate section, we will present the solution of our study. This solution is detailed and divided into three parts: design, implementation and results obtained. Finally Section 6 concludes our article.

\section{$2 \quad$ IoT applications}

The IoT holds some promise for reducing energy consumption that can help us streamline our energy needs.

With the Internet of Things, mobile devices can communicate over wireless networks. As a result, these processes are increasingly automated by reporting all types of information that can be used for optimization. In addition, there is a real-time aspect that allows the supply to better adapt to the demand. All of this makes it possible to process large amounts of data to finely model user behaviour. The producer can then deduce future demand. All these elements have a significant effect on storage, energy consumption and security.

An optimized design and more efficient electronic components are needed to minimize the energy consumption of connected objects. European companies have developed very low power technologies, which is also a discriminating factor for Asian or American manufacturers. But connected objects have to communicate, which consumes energy. Data transmission via $3 \mathrm{G}$ or $4 \mathrm{G}$ networks is costly. As a result, special 
networks such as LoRa or Sigfox have emerged, allowing devices to communicate with each other with less energy consumption.

The possibilities exposed by the Internet of Things allow the improvement of a huge number of applications. Many areas can be impacted by improving our technology: at home, on the road, at work, in case of illness.

Several application areas are affected by the IoT; among these main areas, we cite security, the field of transport, the environment and infrastructure and public services...

\section{Other technologies}

There are several types of connected object technology among these technologies:

\subsection{Radio frequency identification (RFID)}

First of all, it is essential to know that there are two RFID technologies, active and passive RFID. What distinguishes them is the object that emits the signal.

In passive RFID, the tag is a simple antenna activated within the range of a transmitter. This is typical, what you find in many anti-theft systems in stores. The tag you have to cut is an RFID tag, and the security gates are transmitters. It's also a system that many users use daily for access control.

As far as active RFID is concerned, it is about objects, including a tag and emitting the signal near another RFID object. The interest of this technology is to extend the range of the signal to a few meters. Let us make an application that everyone knows, your electronic toll tag. It consists of a battery and an antenna. When you pass near a barrier, your badge sends its identifier.

It is essential to understand that RFID is a technology that is not self-sufficient. It must absolutely be coupled with another technology to collect and store data.

\subsection{WSN}

Wireless Sensor Networks or WSN is a set of dispersed sensors their role is to monitor and record the physical conditions of the environment as well as to organize the collected data. WSNs are autonomous devices that can associate with RFID chips to manage objects' movements, know their temperature and movements, and use their different capacities [14].

Wireless sensor networks allow network topologies to communicate in multi-hop. The latest advances in low power integrated circuits and wireless communications have enabled these low power devices to achieve high-efficiency thanks to WSN [15].

They are mainly used in several areas, such as cold chain logistics or packaging methods [16] on the one hand, and on the other hand, they are used to maintain and monitor systems.

\subsection{Middleware}

It is the intermediary between different applications that helps developers to communicate. It has become an important architectural element in supporting distributed 
applications. Its role is to present a model to software users to reduce and disappear any sort of problem. It became popular in the 1980 s due to its central role in simplifying and integrating traditional technologies into the news. It helps to facilitate the development of non-centralized IT services. Middleware is layered between network operating systems and application components [17]. The distributed IoT infrastructure with many heterogeneous devices must simplify the tasks of new applications so that this technology is a solution for IoT applications [18]. For example, GSN is a platform for middleware that provides a scalable infrastructure to integrate heterogeneous sensor network technologies. Middleware architectures take a service-based view to support different network topologies.

\subsection{Cloud computing}

C. C. is an on-demand access model that corresponds to access to IT services whose purpose is to share resources such as "computers, networks, servers, storage, applications, services, software". It can also be defined as a tool for storing and accessing data via an Internet intermediary. There are three different CC services: IaaS, PaaS and SaaS IoT application needs a powerful medium to store this data, high speed of processing the stored data, analyze it, and predict results to make decisions across high-speed networks. Among the advantages of the CC offers a possibility of the "back end" to analyze the data in real-time without human intervention.

\subsection{Other technology}

In this part we present other IOT technologies by comparing their strong points with their Weaknesses (Table 1):

Table 1. The table of different IOT technologies

\begin{tabular}{|l|l|l|l|}
\hline Technology & \multicolumn{1}{|c|}{ Strong Points } & \multicolumn{1}{c|}{ Weak Points } & \multicolumn{1}{c|}{ Type of Use Case } \\
\hline LoRa & $\begin{array}{l}\text { Low energy consumption } \\
\text { Long-range } \\
\text { Operable standard }\end{array}$ & $\begin{array}{l}\text { Global coverage still low } \\
\text { Data size and volume }\end{array}$ & $\begin{array}{l}\text { Reading of energy meters } \\
\text { Sending specific geolocation } \\
\text { information }\end{array}$ \\
\hline Sigfox & $\begin{array}{l}\text { Low energy consumption } \\
\text { Long-range } \\
\text { Single operator }\end{array}$ & $\begin{array}{l}\text { Global coverage still low } \\
\text { Data size and volume }\end{array}$ & $\begin{array}{l}\text { Reading of energy meters } \\
\text { Sending one-off information } \\
\text { geolocation }\end{array}$ \\
\hline M2M & $\begin{array}{l}\text { Extensive global } \\
\text { coverage }\end{array}$ & $\begin{array}{l}\text { Energy consumption } \\
\text { Scope }\end{array}$ & $\begin{array}{l}\text { Payment terminals } \\
\text { Application with large volumes } \\
\text { of data or sending incident data }\end{array}$ \\
\hline RFID & $\begin{array}{l}\text { No wave emission } \\
\text { No need for energy }\end{array}$ & $\begin{array}{l}\text { Low range } \\
\text { Need for gateway }\end{array}$ & $\begin{array}{l}\text { Zone geolocation } \\
\text { Identification (access control, } \\
\text { authorization, ...) }\end{array}$ \\
\hline Bluetooth & $\begin{array}{l}\text { Message size and volume } \\
\text { Debit }\end{array}$ & $\begin{array}{l}\text { Low range } \\
\text { Need for a gateway }\end{array}$ & \\
\hline
\end{tabular}




\section{$4 \quad$ Related work}

In this section, we describe recent research related to IoT energy reduction methods.

Several recent research has been conducted for the reduction of IoT energy consumption [19-20]. In order to manage the massive amount of data and at the same time have reasonable energy consumption, the most effective solution is from L. Mottola and G. P. Picco, who presented an application approach focused on flow management for IoT services. This solution focuses on measurement scenarios that use a centralized approach, where the nodes send all their measurements to the base. The latter stores them, usually in a database, and allows users to retrieve and process this information later [21]. This single-hop mode can be used if the sensors are close to the base. However, for more extensive sensor networks, it becomes necessary to employ multi-hop communication. The nodes are linked directly to the base, which increases the possibility of having link problems when all nodes are connected in real-time.

Previous work on scheduling algorithms has focused on decreasing the schedule length [22-23] or on distributed implementation [23-25].

A heuristic algorithm for programming several independent segments that aim to improve the degree of parallel exchange and transmission has been proposed [26].

A cross-type optimization protocol that makes energy-efficient and minimizes delay in WSN networks has been proposed [27].

Other technologies have been achieved that are described in the previous chapter unfold to minimize consumption.

\section{Solution}

\subsection{Conception}

There are several solutions to reduce the energy consumption of connected objects: a continuous data transfer has been made in a desire for hyper-connectivity. However, in many cases, a device only needs to be connected to the network occasionally, as is the case for all devices operating in automation.

Connected objects are built around two main families of devices that interact with each other:

Nodes: These devices are the most common in the network. They are usually equipped with a low-powered processor, a wireless communication interface and a limited memory. They are the ones that carry out the measurements in the field, thanks to the sensors they carry.

Bases: These are much more powerful devices, but they do not have sensors. They are generally limited in number in the network. They are used as centralized collection points receiving the measurements acquired by the nodes, as intermediaries between two sensor networks, or with another network (the Internet, in particular) or as points of interaction with users.

After analyzing existing models [28-29], we propose a fully distributed trust model. We did not apply the direct linkage rules with the base, but we introduced a new rule 
according to which nodes communicate with each other and exchange data: "Nodes A and $\mathrm{B}$ send to $\mathrm{C}$, then $\mathrm{C}$ and $\mathrm{D}$ and $\mathrm{E}$ also send $\mathrm{F}$ to go immediately" Bottom (Figure 2).

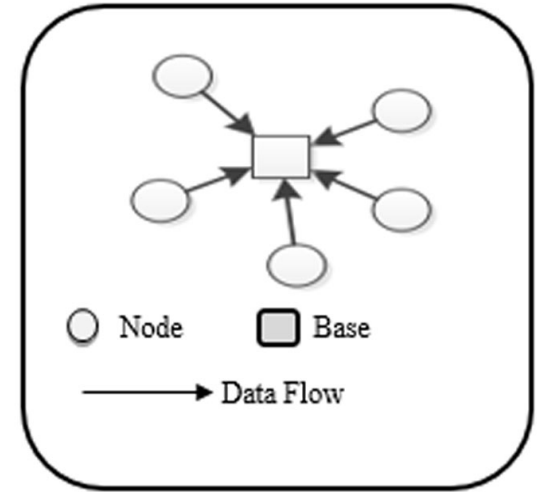

Fig. 1. Centralized collection

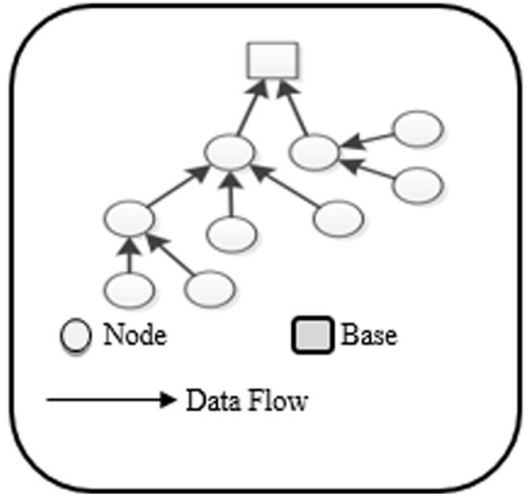

Fig. 2. Distributed colletion

The problem of data transmission: a single bit sent can sometimes consume as much energy as the execution of a thousand instructions by the processor. In order to reduce energy consumption, we have the choice between several solutions:

- Time slots: in this solution, the nodes are only allowed to communicate in a regular interval, while the network interface is disabled the rest of the time, which is useful in some cases but not available in most cases (sensitive data).

- Single hop: Each device exchanges information only with devices that are close enough to communicate. However, in order to minimize energy consumption, the range of these links has been dramatically reduced.

- Multi-hop: Each node can serve as an intermediary (the routing role) for other nodes, self-organizing to build a route through which messages pass.

Scenarios often use a centralized approach (Figure 1), where the node sends all information to the base. The latter typically stores it in a database and allows users to retrieve and process the information. In our solution, we want to create a distributed scenario (Figure 2), where each node communicates with another node and sends data to the base node [30-32].

This solution aims to distribute the tasks between the different nodes.

\subsection{Application}

The solution that we have developed in the previous chapter is deployed through a working environment made up of several tools on which the following implementation is based.

The architecture is shown in the following figure: 


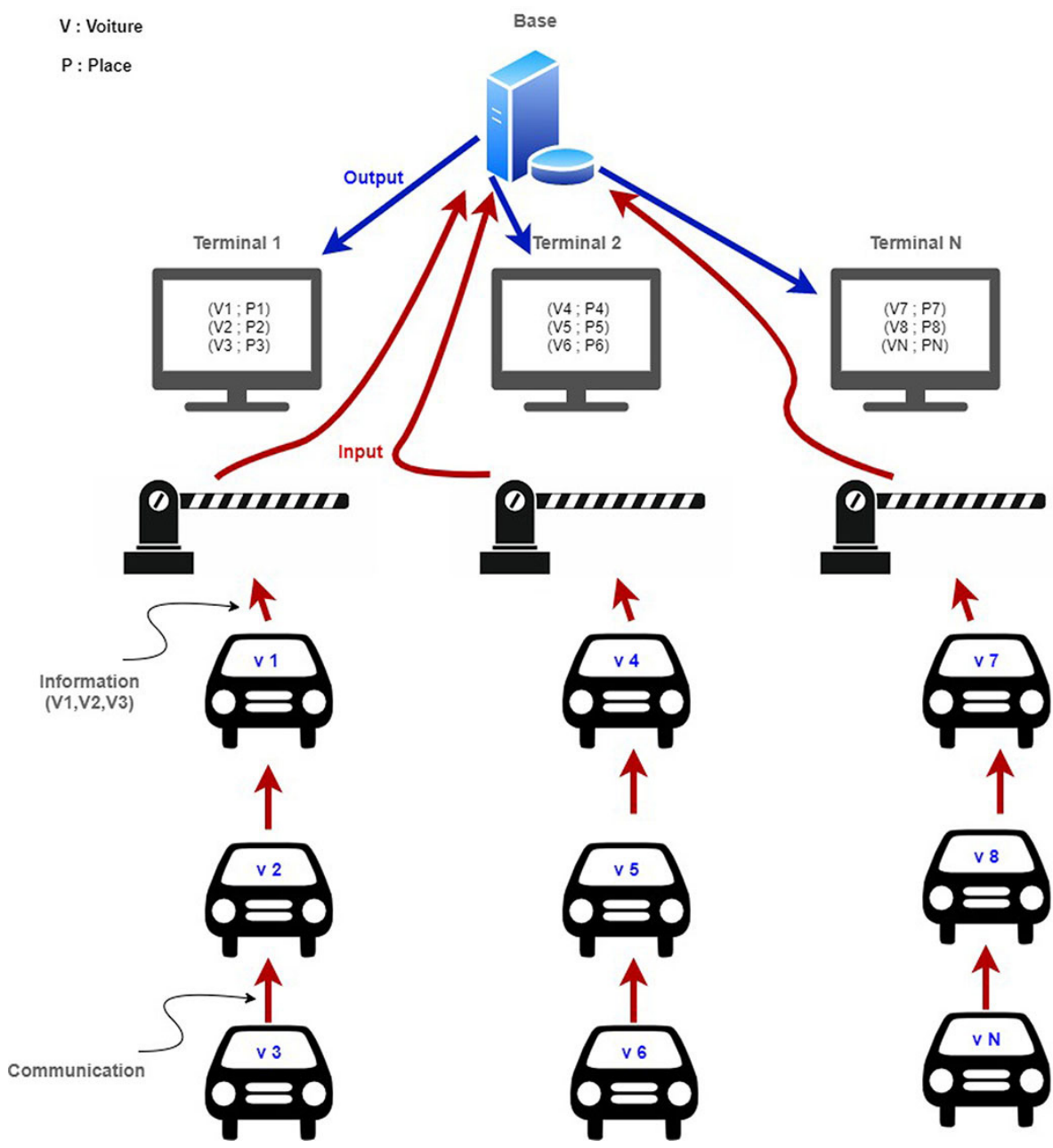

Fig. 3. An application architecture of the solution

After describing and deducing the working solution through Figure 2, we implement our solution in intelligent parking (Figure 3).

To this end, we explain in the following the components of the application architecture that divide our work into three parts:

Part A: (Car/Car):

In this part, we have based on the direct communication between the cars in a hierarchical way so that the last car sends the information to the next car to the first car in one direction

Example: (the first line of cars)

Car 3 sends its ID to Car 2; the latter carry both information and send it to Car 3.

$$
V 1 \rightarrow V 2 \rightarrow V 3
$$


It knows that each car is equipped with a chip that takes on the role of a node/base to communicate with the other cars.

Part B: (Car/Barrier)

This second part has the role of opening the barrier through the communication between the first car and the barrier.

The purpose of the barrier is to process the information.

Example:

Car 1 contains the following information (V1, V2, V3), this information sends to the barrier, and this last one treat this information if it belongs to the identifier of the cars in the parking:

If the case Exact:

Opening in a defined time depends on the number of cars.

If No:

Do not open the barrier by indicating a message on the display board.

Other cases:

If the information sent is:

$$
(V 1, V 2, V 3) \rightarrow \text { Barrier1 }
$$

Also, the second car does not correspond to the parking, and the opening only is for $\mathrm{V} 1$ and $\mathrm{V} 3$.

Part C: (Barrier/Base)

The last part of our solution is to make a communication between the barrier and the base. After processing the data of the cars on the barrier, this information is transferred to the base so that they can be stored in the Cloud and analyzed to make predictions in the long term as well as display the states.

A message will be displayed on the terminals for each line of entry that will guide the cars to their places in the parking lot.

\subsection{Implementation}

In this part, we implement our solution of figures two and three (Node/Base) to be able to compare and analyze the results and demonstrate the degree of success of our solution.

For this, we need the:

Hardware:

We use as hardware to implement the two solutions:-Arduino board, Servo Motor, LCD screen, Multimeter, Board, Cable, Potentiometer, and Resistor. 


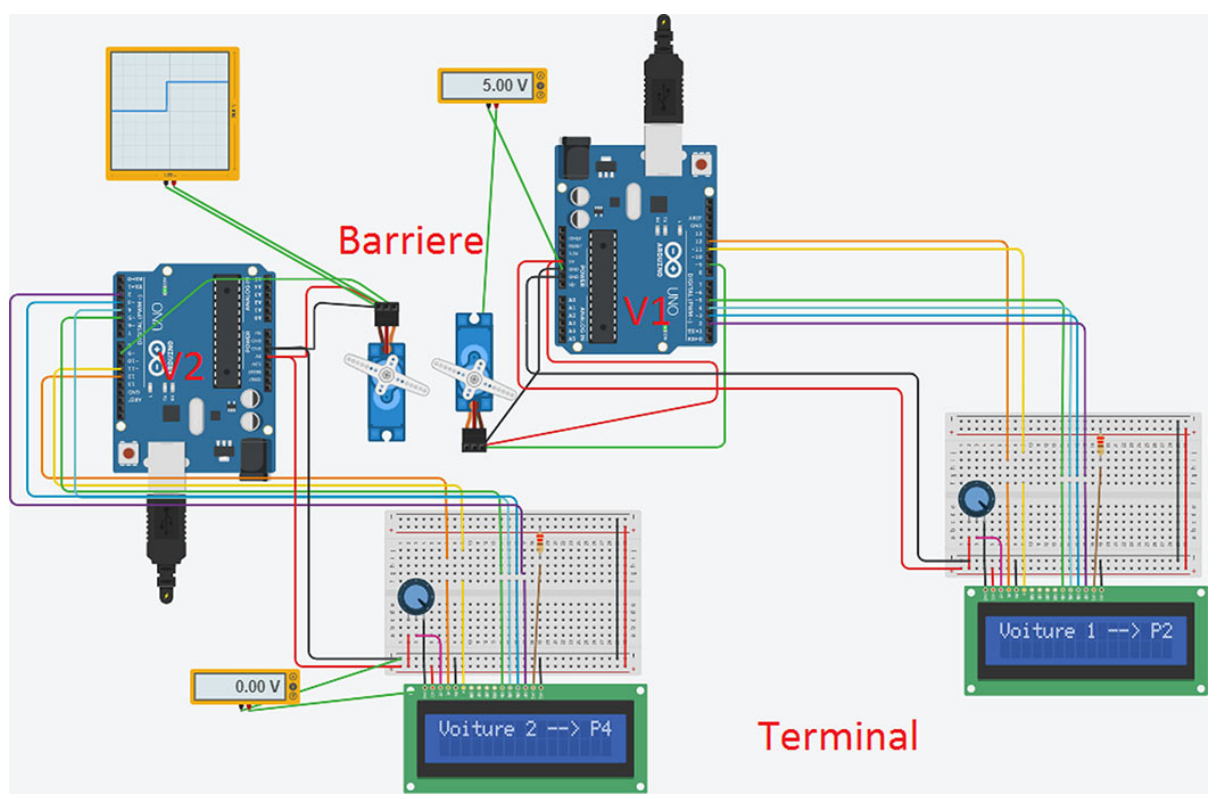

Fig. 4. Simulation centralized collection

- Figure 3 is the result of implementing the first solution based on the connection between the nodes and the bases, as already demonstrated in Figure 1.

- In our demonstration, we have taken the case of our application of intelligent parking on the one hand.

On the other hand, we have chosen only one part of the intelligent parking to demonstrate the result of the energy reduction.

- Barriers: Servo-Motor, - Car: Arduino, - Terminal: LCD screen

In this solution, for the car (V1/Node) to enter the parking lot, it must communicate with the base. The latter sends these coordinates to terminal T1, displaying them and transmitting them to barrier B1 to open, as shown in the diagram in Figure 4.

We have equipped our simulation with Multimeter and Potentiometer to calculate the current passing during the communication between Node/base, and we have been able to notice that out of the sending of information, we can consume a little bit of $5 \mathrm{~V}$ (Table 2).

Table 2. Representative table of the centralized result collection

\begin{tabular}{|l|l|l|}
\hline & \multicolumn{1}{|c|}{ Terminal } & \multicolumn{1}{c|}{ Barrier } \\
\hline Car 1 & $5 \mathrm{~V}$ & $5 \mathrm{~V}$ \\
\hline Car 2 & $5 \mathrm{~V}$ & $5 \mathrm{~V}$ \\
\hline
\end{tabular}

The equation is written as follow:

$$
\mathrm{E}=T V 1+T V 2+B V 1+B V 2
$$




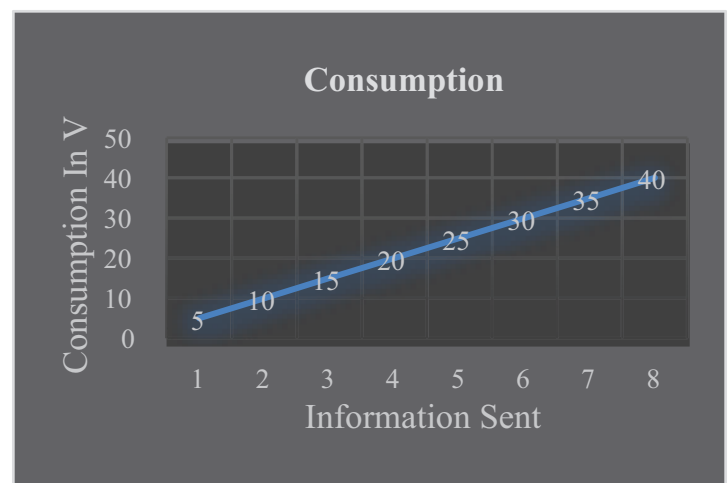

Fig. 5. Graph simulation centralized

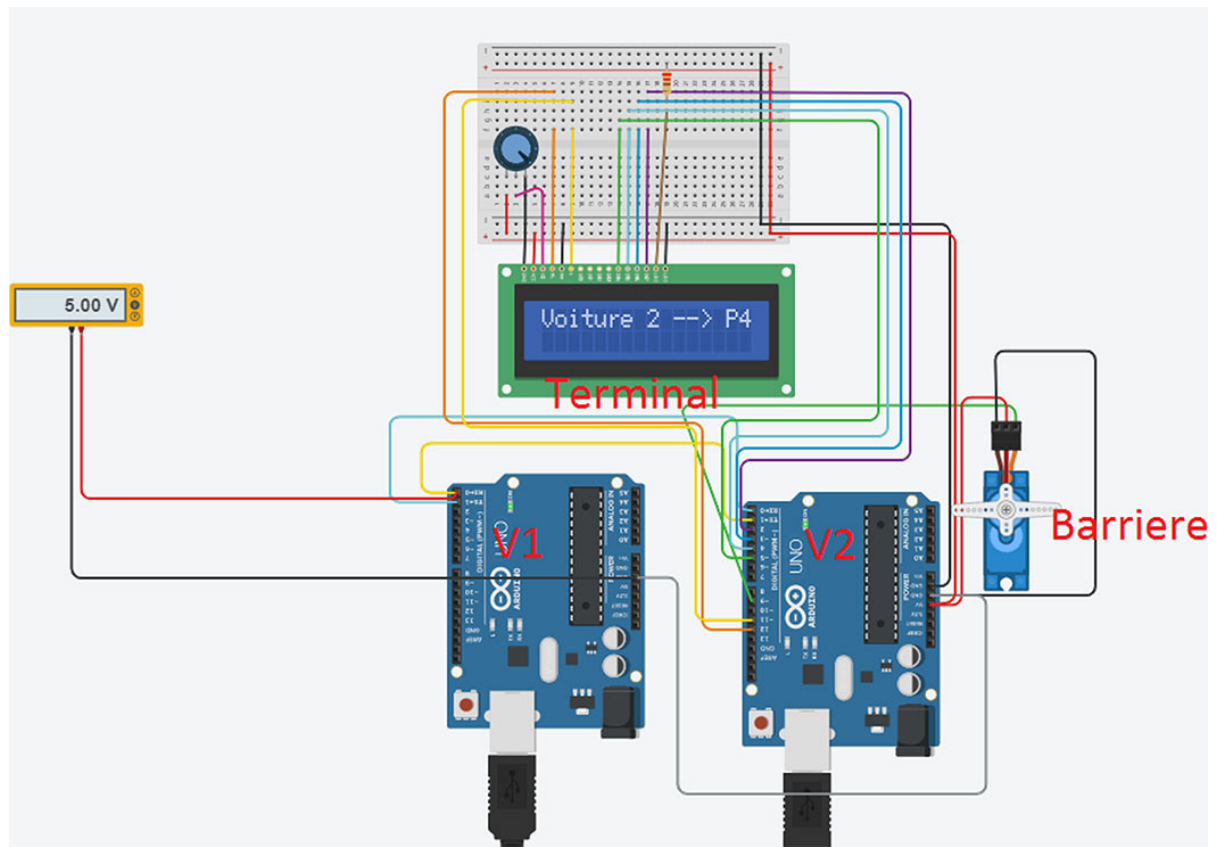

Fig. 6. Simulation distributed collection

Our solution in Figure 6 aims to link the nodes directly between them, not needing an intermediary that is the base. In this case, the car (V2/Node) in order to enter the parking lot, must send its cords to V1. This last one is going to send the cords of the two cars to the terminal T1. Similarly, the terminal sends the previous information to barrier B1 to open, as the diagram shows it. 
Table 3. Representative table of the result distributed collection

\begin{tabular}{|l|l|l|}
\hline & \multicolumn{1}{|c|}{ Terminal } & \multicolumn{1}{|c|}{ Barrier } \\
\hline Car 1 & $5 \mathrm{~V}$ & $5 \mathrm{~V}$ \\
\hline Car 2 & $5 \mathrm{~V}$ & - \\
\hline
\end{tabular}

The equation is written as follow:

$$
\mathrm{E}=T V 1+T V 2+B V 1
$$

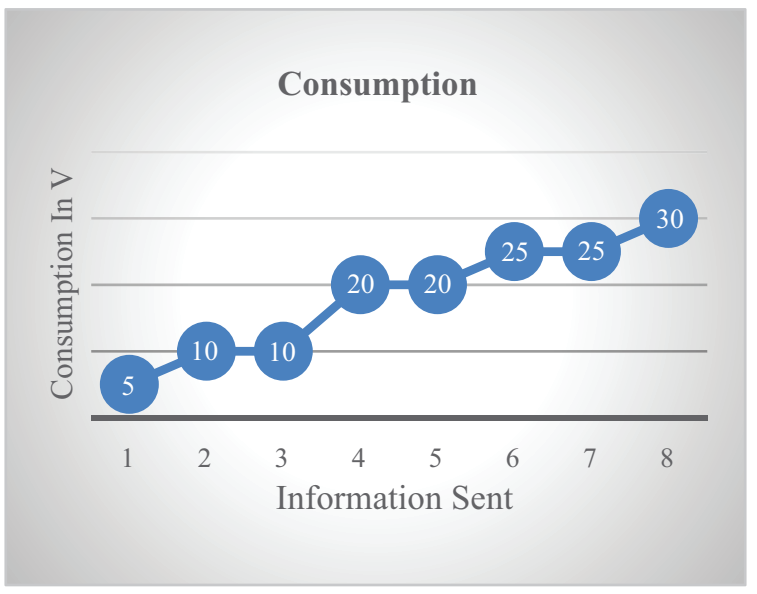

Fig. 7. Graph simulation distributed

We have equipped our simulation of Multimeter and Potentiometer to calculate the current passing during the communication between Node/base, and we concluded with the demonstrative table (Table 3).

In this study of the two solutions as well as the comparison of the results obtained from the two figures 5 and 7 , we were able to prove using the extracted results. This solution guarantees an increasing energy reduction with the increase in the number of devices used in a project.

\section{Conclusion}

The world has been attacked by a new phenomenon: "connected objects". These objects facilitate daily tasks and help humanity to solve their problems. They are objects that use the Internet to improve their functioning via networks, often the technical evolution of an existing object. Despite this evolution, there remain many problems that this kind through these objects, such as energy consumption.

Implementing an energy optimization system remains a challenge in the face of the development of this technology. That is why this paper was devoted to identifying the areas of IoT application and explaining the progress they were experiencing. 
Then, our solution provides a low power consumption solution for setting up a system. This system can be adapted to the customer's needs, for example, parking, city, house. Our approach provides solutions to the various problems posed by the Internet of Things, linked to the consumption and volume of data to the heterogeneity of objects. However, the solution aims to give benefit to these connected objects. Indeed, after the study and research, our solution is implemented so that it is applicable. Finally, this work is a step towards an Internet of things responding to all the problems.

\section{$7 \quad$ References}

[1] M. Weiser, "The computer for the 21st century" Scientific American, vol. 256, no. 3, 1991. https://doi.org/10.1038/scientificamerican0991-94

[2] L. Atzori, A. Iera, and G. Morabito, "The internet of things: a survey," Computer Networks, vol. 54, no. 15, pp. 2787-2805, 2010. https://doi.org/10.1016/j.comnet.2010.05.010

[3] E. Fleisch, "What is the internet of things? An economic perspective," Tech. Rep., Auto-ID Labs, 2010.

[4] National Intelligence Council, "Disruptive civil technologies - six technologies with potential impacts on us interests out to 2025," Conference Report CR 2008-07, 2008.

[5] I. Roxin, and A. Bouchereau, "Ecosystème de l'Internet des Objets", dans Bouhaï N. et Saleh I., (dir.) "Internet des objets : Evolutions et Innovations", ISTE Editions Londres, Mai 2017.

[6] M. R. Palattella, N. Accettura, X. Vilajosana et al., "Standardized protocol stack for the internet of (important) things", IEEE Communications Surveys and Tutorials, vol. 15, no. 3, pp. 1389-1406, 2013. https://doi.org/10.1109/SURV.2012.111412.00158

[7] S. Tozlu, M. Senel, W. Mao, and A. Keshavarzian, "Wi-Fi enabled sensors for internet of things: a practical approach," IEEE Communications Magazine, vol. 50, no. 6, pp. 134-143, 2012. https://doi.org/10.1109/MCOM.2012.6211498

[8] N. Bui, A. P. Castellani, P. Casari, and M. Zorzi, "The internet of energy: a web-enabled smart grid system," IEEE Network, vol. 26, no. 4, pp. 39-45, 2012. https://doi.org/10.1109/ MNET.2012.6246751

[9] X. Fang, S. Misra, G. Xue, and D. Yang, "Smart grid - the new and improved power grid: a survey," IEEE Communications Surveys and Tutorials, vol. 14, no. 4, pp. 944-980, 2012. https://doi.org/10.1109/SURV.2011.101911.00087

[10] INFSO D.4 Networked Enterprise \& RFID. (Sep. 2008). Internet of Things in 2020-ARoadmap for the Future. [Online]. Available: https://www. smart-systems-integration.org/public/documents/publications/Internet-ofThings in 2020 EC-EPoSS_Workshop_Report 2008 v3.pdf

[11] I. Mavrommati, and A. Kameas, "The evolution of objects into hyper-objects: will it be mostly harmless?", Personal and Ubiquitous Computing. 2003. Disponible sur : https://doi. org/10.1007/s00779-003-0223-1

[12] M. Weiser, "Hot Topics: Ubiquitous Computing", IEEE Computer, octobre 1993. https://doi. org $/ 10.1109 / 2.237456$

[13] M. Moutaib, "Internet of things: Energy Consumption and Data Storage", Procedia Computer Science, Volume 175, 2020, 609-614. https://doi.org/10.1016/j.procs.2020.07.088

[14] Atzori, L., Iera, A., and Morabito, G. (2010). The Internet of Things: A survey. Computer Networks, 54(1), 52787-52805. https://doi.org/10.1016/j.comnet.2010.05.010 
[15] Gubbi, J., Buyya, R., Marusic, S., and Palaniswami, M. (2013). Internet of Things (IoT): A vision, architectural elements, and future directions. Future Generation Computer Systems, 29(7), 1645-1660. https://doi.org/10.1016/j.future.2013.01.010

[16] Hsueh, C.-F., and Chang, M.-S. (2010). A model for intelligent transportation of perishable products. International Journal of Intelligent Transportation Systems Research, 8(1), 36-41. https://doi.org/10.1007/s13177-009-0004-y

[17] W. Emmerich, Engineering Distributed Objects. John Wiley \& Sons, Apr. 2000. https://doi. org/10.1007/3-540-45254-0

[18] In Lee, Kyoochun Lee. "The Internet of Things (IoT): Applications, investments, and challenges for enterprises", Business Horizons, 2015. https://doi.org/10.1016/j. bushor.2015.03.008

[19] Benjamin Billet, Système de gestion de flux pour l'Internet des objets intelligents. Calcul parallèle, distribué ET partagé [cs.DC]. Université de Versailles-Saint Quentin en Yvelines, 2015.

[20] G. Sun, V. Chang, M. Ramachandran, Z. Sun, G. Li, H. Yu and D. Liao, Efficient Location Privacy Algorithm for Internet of Things (IoT) Services and Applications, Journal of Network and Computer Applications, https://doi.org/10.1016/j.jnca.2016.10.011

[21] L. Mottola and G. P. Picco, "Programming wireless sensor networks: Fundamental concepts and state of the art," ACM Computing Survey, vol. 43, no. 3, 2011. https://doi. org/10.1145/1922649.1922656

[22] X. H. Xu, X.Y. Li, X. Mao, et al., "A Delay-Efficient Algorithm for Data Aggregation in Multihop Wireless Sensor Networks", IEEE Transactions on Parallel and Distributed Systems, vol. 22, no. 1, 2011, pp.163-175. https://doi.org/10.1109/TPDS.2010.80

[23] S. Wang and Z. Chen, "LCM: A Link-Aware Clustering Mechanism for Energy-Efficient Routing in Wireless Sensor Networks", IEEE Sensors Journal, vol. 13, no. 2, 2013, pp. 728-736. https://doi.org/10.1109/JSEN.2012.2225423

[24] M. Martalo, C. Buratti, G. Ferrari, et al., "Clustered IEEE 802.15. 4 Sensor Networks with Data Aggregation: Energy Consumption and Probability of Error", IEEE Wireless Communications Letters, vol. 2, no. 1, 2013, pp. 70-73. https://doi.org/10.1109/ WCL.2012.112012.120644

[25] A. Tarik, and Y. Farhaoui, (2019) Recommender System for Orientation Student BDNT 2019: Big Data and Networks Technologies, pp. 367-370. https://doi. org/10.1007/978-3-030-23672-4_27

[26] S. Gandham, Y. Zhang, and Q. Huang, "Distributed time-optimal scheduling for convergecast in wireless sensor networks," Computer Networks, vol. 52, no. 3, 2008, pp. 610-629. https://doi.org/10.1016/j.comnet.2007.10.011

[27] L. Shi and A.O. Fapojuwo, "TDMA Scheduling with Optimized Energy Efficiency and Minimum Delay in Clustered Wireless Sensor Networks", IEEE Transactions on Mobile Computing, vol. 9, no. 7, 2010, pp. 927-940. https://doi.org/10.1109/TMC.2010.42

[28] M. Omar, Y. Challal, and A. Bouabdallah, "Certification-based trust models in mobile ad hoc networks : A survey and taxonomy", Journal of Network and Computer Applications (Elsevier), Volume 35, Issue 1, January 2012, Pages 268-286. https://doi.org/10.1016/j. inca.2011.08.008

[29] M. Omar, Y. Challal, A. and Bouabdallah, "Reliable and fully distributed trust model for mobile ad hoc networks", Computers and Security (Elsevier), vol. 28, num. 3-4, pp. 199214, 2009. https://doi.org/10.1016/j.cose.2008.11.009

[30] Z. Baharum, A. S. Sabudin, E. M. M. Yusof, and N. A. Ahmad, (2021). Mobile-Based Application: The Designation of Energy Saving Smart Light System for Monitoring and Controlling. International Journal of Interactive Mobile Technologies (iJIM), 15(18), 90. https://doi.org/10.3991/ijim.v15i18.24733 
[31] M. G. Maru, C. C. Pikirang, S. Setiawan, E. Z. O. Oroh, and N. Pelenkahu, (2021). The Internet Use for Autonomous Learning During COVID-19 Pandemic and its Hindrances. International Journal of Interactive Mobile Technologies (iJIM), 15(18), 65. https://doi. org/10.3991/ijim.v15i18.24553

[32] Istiyowati, L. S., Syahrial, Z., and Muslim, S. (2021). Readiness for the Implementation of Ubiquitous Learning in Programming Course in Higher Education. International Journal of Interactive Mobile Technologies (iJIM), 15(18), 33. https://doi.org/10.3991/ijim. $\underline{\mathrm{v} 15 \mathrm{i} 18.24547}$

\section{Authors}

Mohammed Moutaib, Department of Computer Science, Faculty of Science, University of Moulay Ismail, Meknes, Morocco (Email: mohammed.93@live.fr).

Tarik Ahajjam, Department of Computer Science, Faculty of Science and Technics, University of Moulay Ismail, Errachidia, Morocco (Email: ahajjam.tarik@gmail.com).

Mohammed Fattah, Department of Computer Science, Faculty of Science, University of Moulay Ismail, Meknes, Morocco (Email: fattahm@gmail.com).

Yousef Farhaoui, Department of Computer Science, Faculty of Science and Technics, University of Moulay Ismail, Errachidia, Morocco (Email: y.farhaoui@,fste. umi.ac.ma).

Badraddine Aghoutane, Department of Computer Science, Faculty of Science, University of Moulay Ismail, Meknes, Morocco (Email: b.aghoutane@umi.ac.ma).

Moulhime El Bekkali, Laboratory of IASSE, University of Sidi Mohamed Ben Abdellah, Fez, Morocco (Email: moulhime.elbekkali@usmba.ac.ma).

Article submitted 2021-09-17. Resubmitted 2021-10-11. Final acceptance 2021-10-12. Final version published as submitted by the authors. 